\title{
Mentoring System: Student Leadership Programme
}

\author{
Hiral Makwana \\ Information Technology \\ Shah and Anchor Kutchhi Engineering College \\ Mumbai, India \\ Dr.Kranti Ghag \\ Information technology \\ Shah and Anchor Kutchhi Engineering College \\ Mumbai, India
}

\begin{abstract}
Indian Development Foundation's Student Leadership Programme (SLP) is a 16-hour powerpacked programme. 7 days of life-skill training and the eighth day is for certificate presentation to all the students. The volunteers will be trained in two/ three sessions and they will be called as mentors. The mentors will have to conduct sessions in schools assigned by the IDF. Their main objective is to inculcate leadership skills in the students. Hence, we, SAKEC students, have stepped in to help them. The project includes a web application which will help to connect to colleges for mentors and the schools who wish to be a part of SLP. Sessions can be created and approved. Automatic certificate generation takes place at the end of the sessions. Feedback given by the mentors is analysed to find the positive and negative comments and also the accuracy of the algorithm used for the analysis.
\end{abstract}

Keywords:- IDF, SLP, mentors, session, admin, College Coordinator (CC), School Coordinator (SC)

\section{INTRODUCTION}

Youth is the most significant piece of childhood in one's life. The prospectus/exercises which are significant parts of the educational program leave a vacuum for the advancement of specific characteristics. These characteristics are extremely persuasive in encircling the character of the understudy and requests an equivalent portion of focus and endeavors. Indian Development Foundation (IDF) has been continually supporting and taking a stab at the improvement of the understudies.For this, IDF has built up a preparation program to concentrate on the basics and emotional capacity headway. The course will focus on the improvement of the understudy's attributes which would lead them to be knowledgeable and educated.IDF needs every understudy to be a superior

\author{
Deepika Manche \\ Information Technology \\ Shah and Anchor Kutchhi Engineering College \\ Mumbai, India \\ Sanika More \\ Information Technology \\ Shah and Anchor Kutchhi Engineering College \\ Mumbai, India
}

person. The understudies would be prepared by the corporate representatives/PG understudies and Mentors who have contributed to the SLP. IDF will set up the volunteers who will be called as Mentors. Currently they are operating without any use of technology for communicating with the coordinators and mentors. Thus, students of SAKEC have undertaken this project to help SLP increase its domain.

\section{STRUCTURE}

The project consists of 4 modules- admin, college coordinator, school coordinator and mentor:

\section{Admin Module}

- Will be able to add/remove college and school coordinator

- Will have the right to approve a session

- Will be able to view which college mentors are going to which school

- Can add photos and videos of various sessions

$>$ College Coordinator Module

- Will be able to add/remove mentors

- Will be able to view school details where slots are available for mentoring

- Can apply for the school where they want to send their college students as mentors

\section{School Coordinator Module}

- Will have the option to include meetings

- Will have the option to stamp tutor's participation

$>$ Mentor

- Will be able to add student details

- Will be able to mark student's attendance 


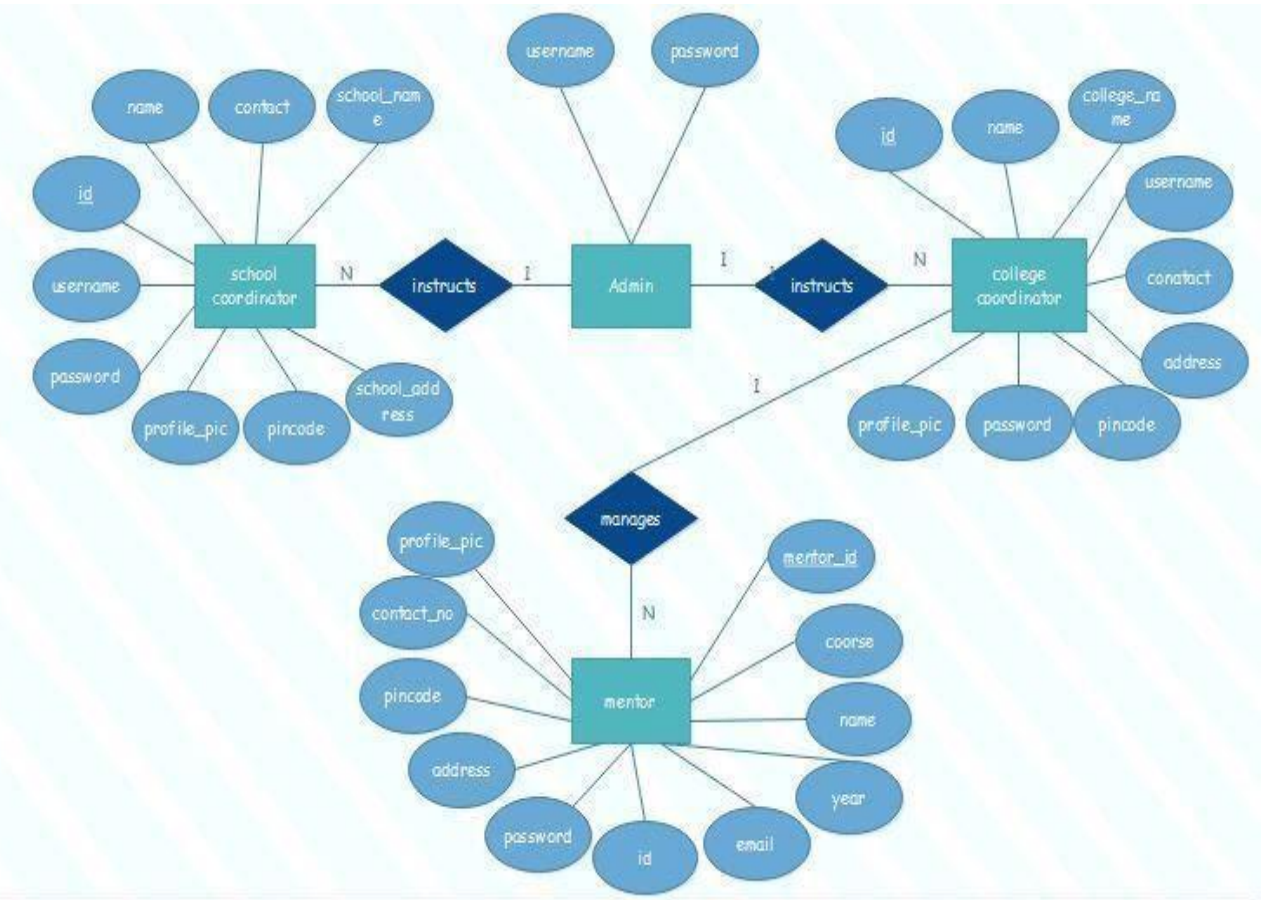

Fig 1

\section{USER INTERFACE}

User interface includes various user-click buttons, input fields for entering data. The main login window consists of taking a username and password, dashboard for viewing the sessions and on-click button for submitting and viewing the details. The interface visualized the features and functionalities listed in this document for this prototype:

Push button for registering and accepting sessions.

$>$ Field for entering details.

\section{A. Hardware interfaces}

The solution makes extensive use of hardware devices including: windows user's computers.

\section{B. Software interfaces}

Other than the hardware interfaces specified, the software requirements are to support windows operating systems.

\section{Communication interfaces}

Internet connection and a web browser are required in order to make use of several functions and to be executed.

\section{SYSTEM LEVELS}

A data flow diagram is one of the most common methods used for graphically representing a data through an information system. A DFD shows what information is provided as input to and output from the system.

A.Level 0 DFD:

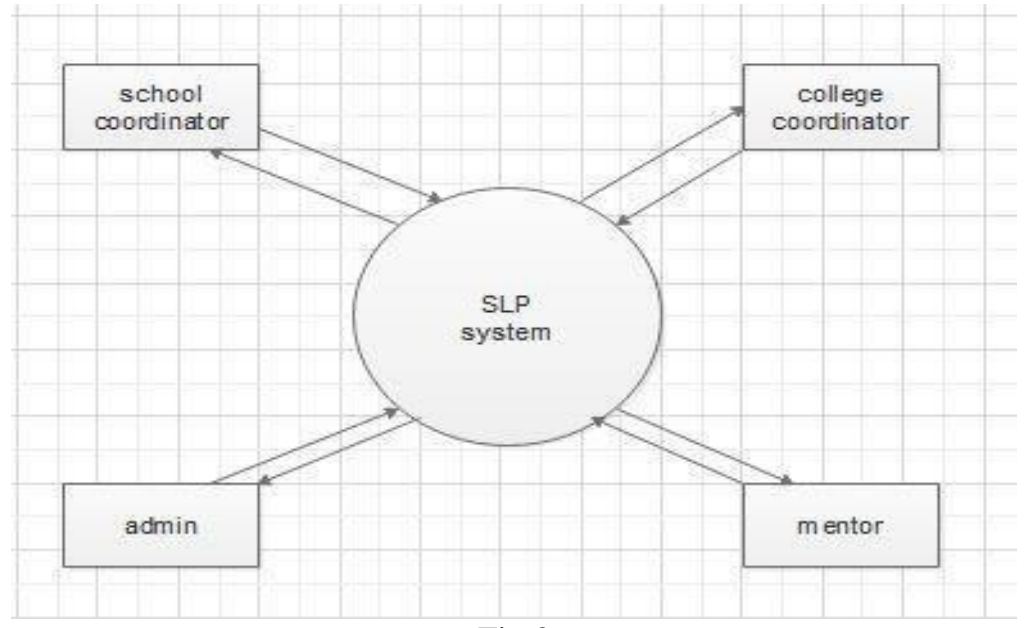

Fig 2 
Level 0 also known as Context level DFD, gives the overview of the whole system in which the external entities like mentor, school coordinator, college coordinator, admin are involved.

B.Level 1 DFD:

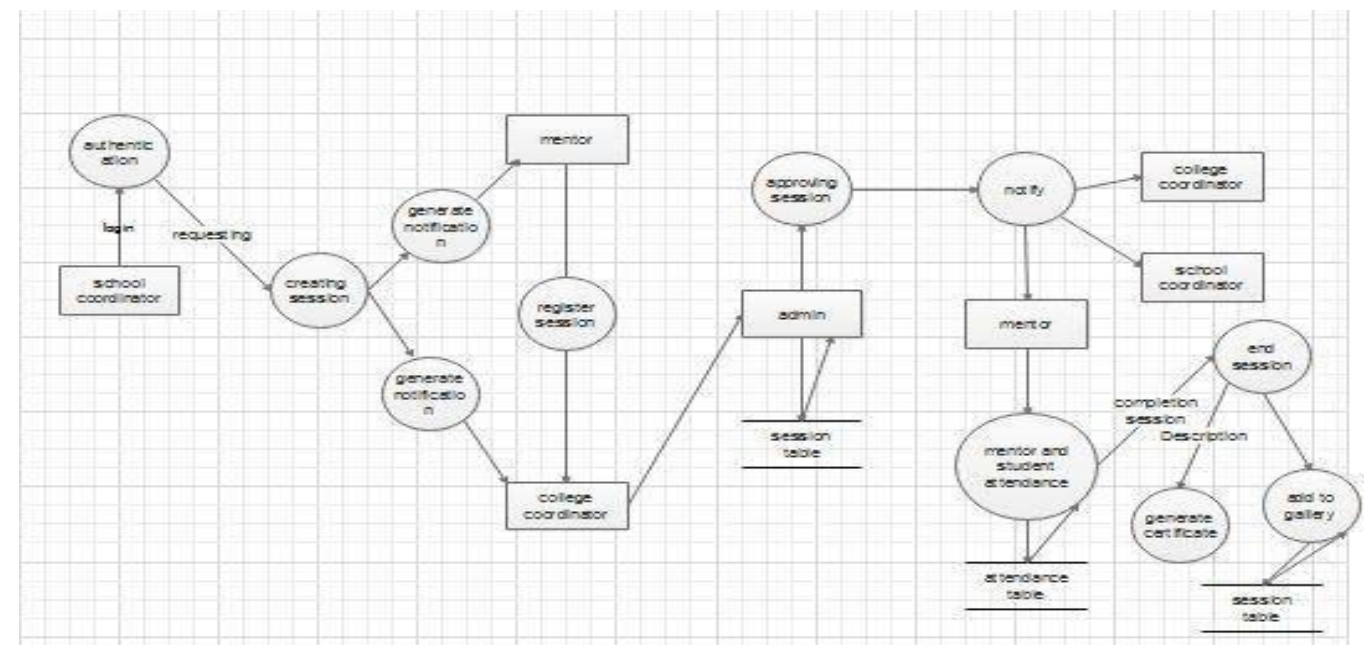

Fig 3

The Figure represents collection of data, induce and deduce model processes and databases of the sessions. It is an elaboration of level 0 DFD. The flow of process from creation to end is illustrated.

C.Level 2 DFD:

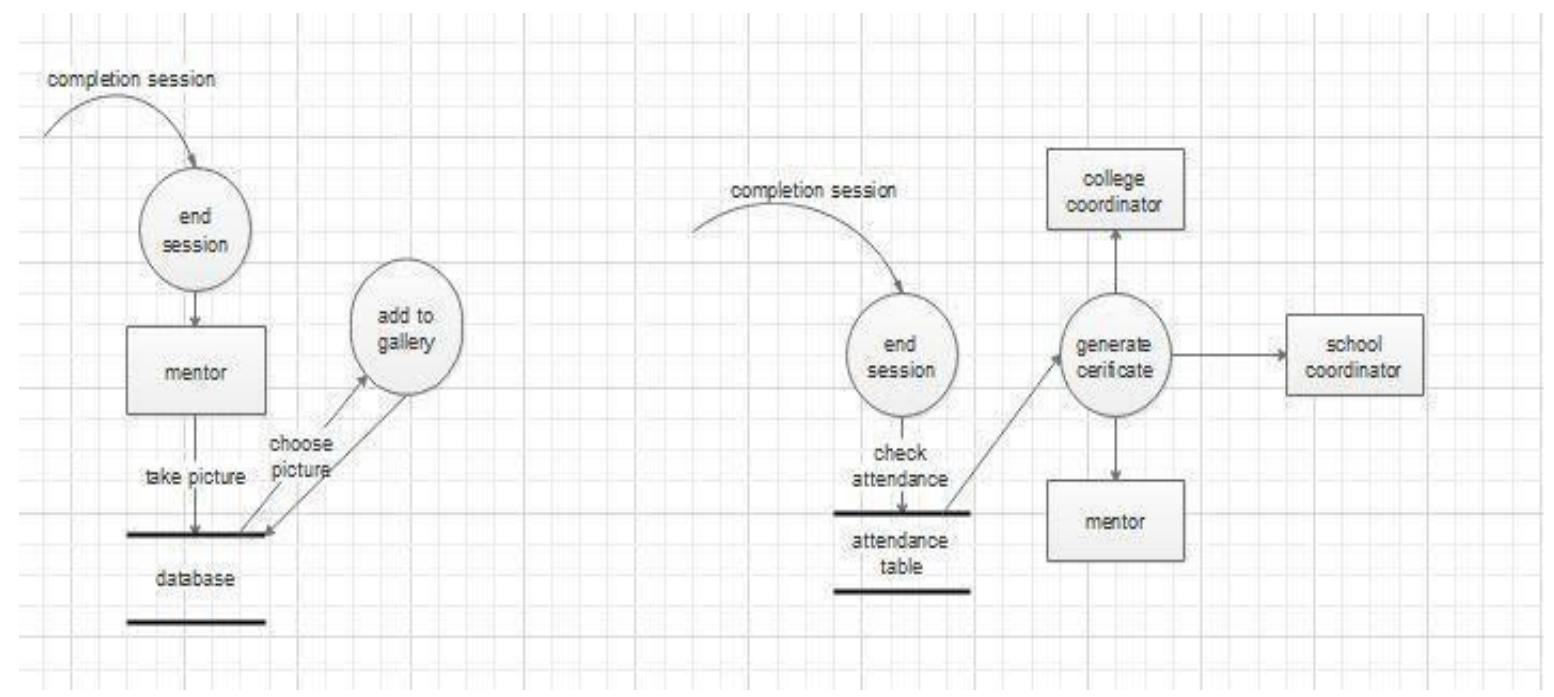

Fig 4

The induced model is further elaborated into two processes- "add to gallery" and "generate certificates" whereas the deduced model is elaborated into "end session" and the model being implemented.

\section{TEXT CLASSIFICATION}

Text classification algorithms are at the heart of a variety of software systems that process text data at scale .In numerous calculations like factual and probabilistic learning strategies, clamor and pointless highlights can adversely influence the general execution. In this way, the end of these highlights is critical. Text feature extraction and pre-taking care of for request computations are
noteworthy.One of the broadly utilized normal language handling undertakings in various business issues is "Text Classification". The objective of text grouping is to consequently order the content records into at least one characterized classifications. A few instances of text arrangement are:

- Understanding audience sentiment from social media,

- Detection of spam and non-spam emails,

- Auto tagging of customer queries, and

- Categorization of news articles into defined topics. 
The classification problem can be classified into below steps:

- Prerequisite and setting up the environment.

- Loading the data set in jupyter.

- Extracting features from text files.

- Running ML algorithms.

- Grid Search for parameter tuning.

- Useful tips and a touch of NLTK.

The different layers used in the process of text classification are as follows:

$>$ Embedding Layer : Word Embeddings give a thick portrayal of words and their relative meanings. They are an improvement over inadequate portrayals utilized in less complex sack of word model representations. Word embeddings can be gained from content information and reused among ventures. They can likewise be learned as a component of fitting a neural system on content data. A word implanting is a class of approaches for speaking to words and archives utilizing a thick vector representation.It necessitates that the information be a whole number encoded, with the goal that each word is spoken to by a one of a kind whole number. This information readiness step can be performed utilizing the Tokenizer API additionally furnished with Keras. The Embedding layer is instated with irregular loads and will get familiar with an inserting for the entirety of the words in the preparation dataset.

> LSTM Layer : Long Short Term Memory systems generally just called "LSTMs" - are an exceptional sort of RNN, equipped for learning long haul conditions. They were presented by Hochreiter and Schmidhuber (1997), and were refined and promoted by numerous individuals in the following work. They work colossally well on a huge assortment of issues, and are currently generally utilized. LSTMs are expressly intended to maintain a strategic distance from the drawn out reliance issue. Recollecting data for extensive stretches of time is for all intents and purposes their default conduct, not something they battle to learn. The way to LSTMs is the phone express, the even line going through the highest point of the diagram. The LSTM has the capacity to evacuate or add data to the phone state, deliberately controlled by structures called entryways.

$>$ Regularization Layer : Regularization is a key part in forestalling overfitting. Likewise, a few methods of regularization can be utilized to lessen model limit while looking after precision, for instance, to drive a portion of the parameters to zero. This may be attractive for diminishing model size or driving down expense of assessment in versatile conditions where processor power is compelled.A portion of the Regularization methods used to address over-fitting and highlight determination are:

- L1 Regularization

- L2 Regularization

A relapse model that utilizes L1 regularization method is called Lasso Regression and model which utilizes L2 is called Ridge Regression. The key differentiation between these techniques is that Lasso recoils the less significant component's coefficient to zero in this way, evacuating some elements out and out. Along these lines, this functions admirably for inclusion in the event that we have a colossal number of highlights.

Dropout Layer : Dropout is a strategy used to keep a model from overfitting. Dropout works by arbitrarily setting the active edges of concealed units (neurons that make up shrouded layers) to 0 at each update of the preparation stage. Dropout can enable a model to sum up by haphazardly setting the yield for an offered neuron to 0 . In setting the yield to 0 , the cost work turns out to be progressively delicate to neighboring neurons changing the manner in which the loads will be refreshed during the procedure of backpropagation. Dropout is a way to deal with regularization in neural systems which helps lessen associated learning among the neurons. Dropout powers a neural system to learn increasingly vigorous highlights that are valuable related to various irregular subsets of different neurons.

> Dense Layer : Thick layer is the standard significantly related neural framework layer. It is commonly typical and as frequently as conceivable used.Thick layers incorporate a fascinating non-linearity property, along these lines they can show any logical limit. Regardless, they are up 'til now limited as in for a comparative data vector we get reliably a comparative yield vector. Thick layer performs procedure on the contribution to give the accompanying yield:

Output $=$ activation $(\operatorname{dot}($ input,kernel $)+$ bias $)$

The output of the analysis is as follows : 


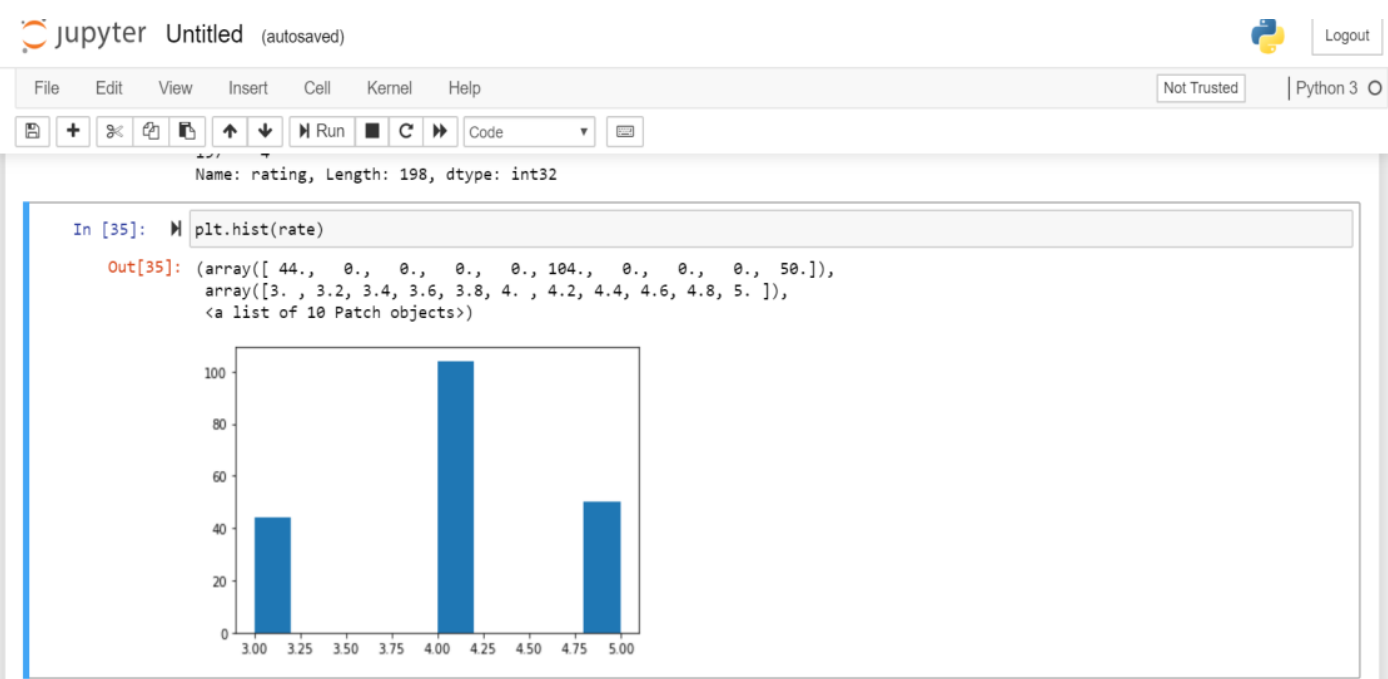

Fig 5

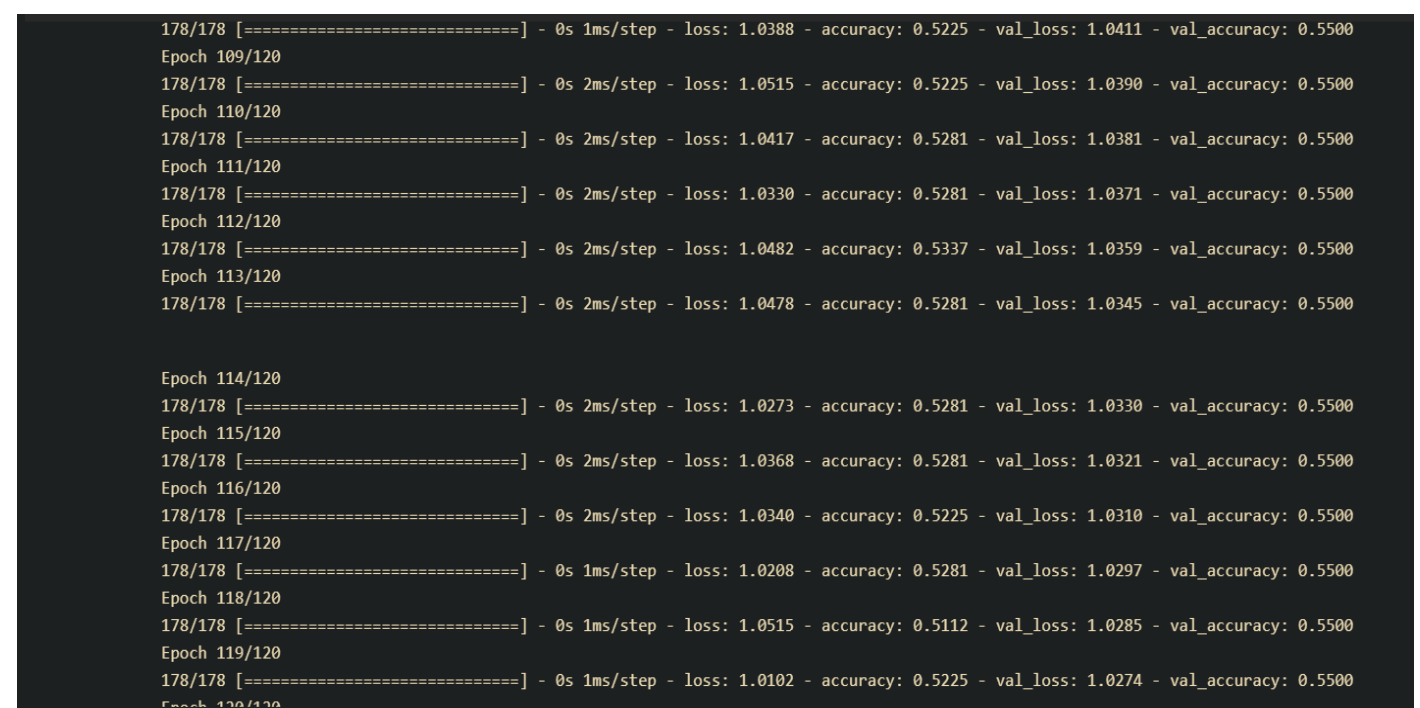

Fig 6
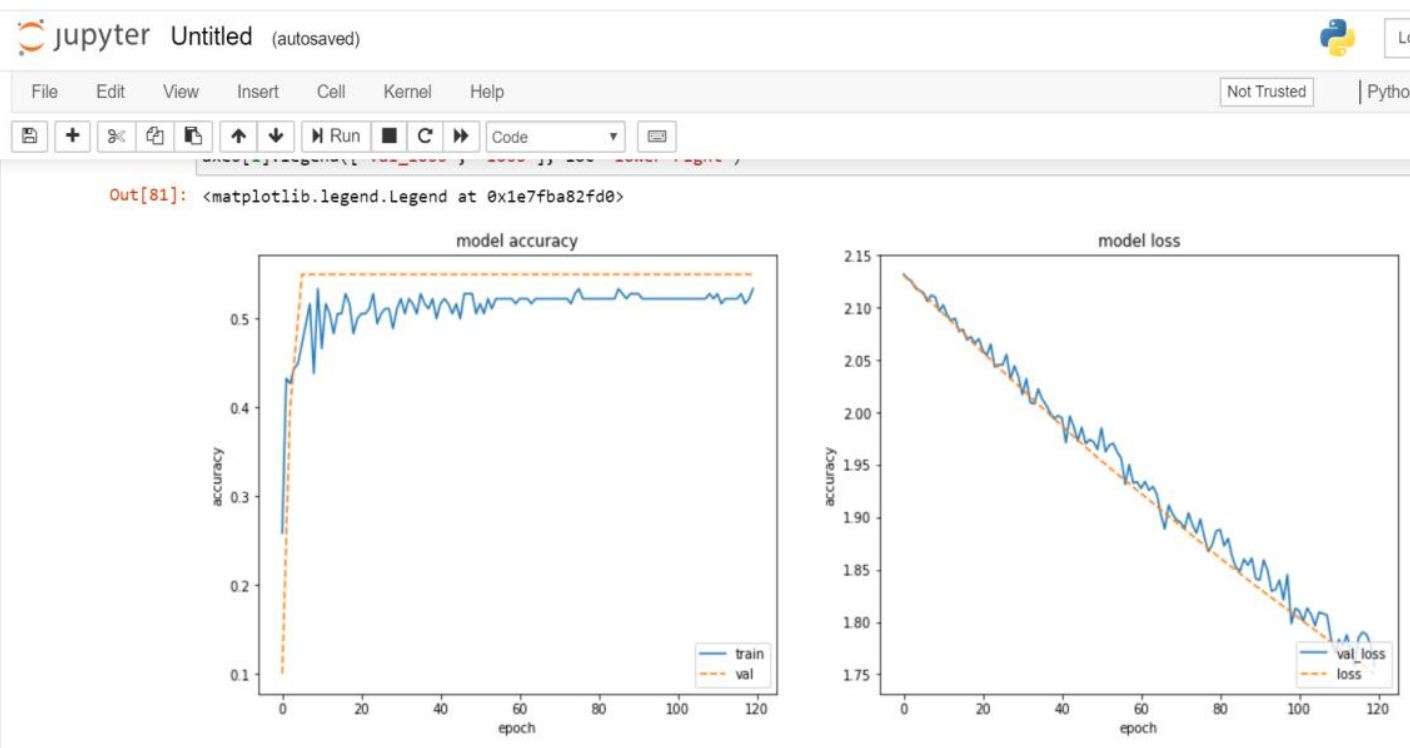

Fig 7 


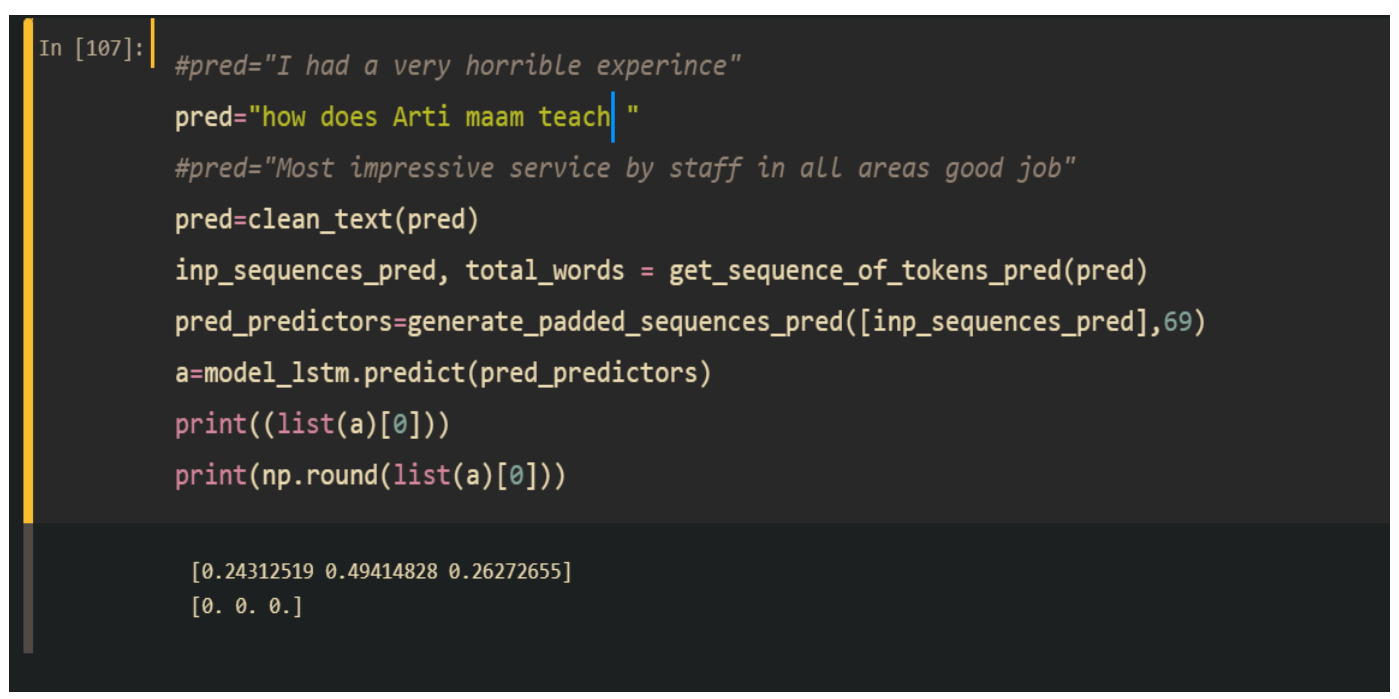

Fig 8

\section{CONCLUSION}

Due to technological assistance, automation of SLP was possible. Adding and accepting sessions becomes convenient. Indian Development Foundation will be able to expand their reach to more schools and colleges. Access of the website can be done anywhere and anytime through the use of mobile phones, laptops, tablets, computers. People will become more aware of the SLP and will participate more frequently. Imparting skills in students becomes easy with the coordination shown among people. Analysis of the feedback was done successfully.

\section{ACKNOWLEDGMENT}

This research was supported by Shah And Anchor Kutchhi Engineering College, Mumbai. We would like to express our gratitude to our project guide Dr. Kranti Ghag for offering her pearls of shrewdness to us over the span of this exploration. We are also immensely grateful to our HOD- Prof. Swati Nadkarni, our Vice Principal- Dr. V.C.Kotak and our principal- Dr. Bhavesh Patel for providing us with the necessary infrastructure.

\section{REFERENCES}

[1]. Erasmus, M., and P. C. Van Der. Westhuizen. Guidelines for the Professional Development of School Principals by Means of a Mentoring System in a Developing Country.

[2]. Mentoring Youth in the Juvenile Justice System. Juvenile Rehabilitation Administration, Mentor Program, 2009.

[3]. Mentoring Students: Creating Success through Caring: Proceedings of the National Conference on Mentoring Minority, Women, Nontraditional, and Handicapped Students, Western Michigan University, April 18 and 19, 1988, Kalamazoo, Michigan. Western Michigan University, 1989.

[4]. Newberger, Julee. Mentoring by Modem. Connect for Kids Guidance for Grown-Ups. Distributed by ERIC Clearinghouse, 2002.
[5]. International Journal of Engineering Research in Africa Vol. 24. Trans Tech Publications Ltd, 2016.

[6]. Liu, Bing. Sentiment Analysis: Mining Opinions, Sentiments, and Emotions. Cambridge University Press, 2020. 